\title{
\begin{tabular}{l|l} 
Mitraries & DSpace@MIT
\end{tabular}
}

\author{
MIT Open Access Articles
}

\section{H I-SELECTED GALAXIES IN THE SLOAN DIGITAL SKY SURVEY. II. THE COLORS OF GAS-RICH GALAXIES}

The MIT Faculty has made this article openly available. Please share how this access benefits you. Your story matters.

Citation: West, Andrew A., Diego A. Garcia-Appadoo, Julianne J. Dalcanton, Mike J. Disney, Constance M. Rockosi, and Zeljko Ivezic. "H I-SELECTED GALAXIES IN THE SLOAN DIGITAL SKY SURVEY. II. THE COLORS OF GAS-RICH GALAXIES.” The Astronomical Journal 138, no. 3 (September 1, 2009): 796-807. (C) 2009 The American Astronomical Society

As Published: http://dx.doi.org/10.1088/0004-6256/138/3/796

Publisher: IOP Publishing

Persistent URL: http://hdl.handle.net/1721.1/93131

Version: Final published version: final published article, as it appeared in a journal, conference proceedings, or other formally published context

Terms of Use: Article is made available in accordance with the publisher's policy and may be subject to US copyright law. Please refer to the publisher's site for terms of use. 


\title{
H I-SELECTED GALAXIES IN THE SLOAN DIGITAL SKY SURVEY. II. THE COLORS OF GAS-RICH
} GALAXIES

\author{
Andrew A. West ${ }^{1,2}$, Diego A. Garcia-Appadoo ${ }^{3,4}$, Julianne J. Dalcanton ${ }^{2}$, Mike J. Disney ${ }^{4}$, Constance M. Rockosi ${ }^{5}$, \\ AND ŽELJKo IVEZIĆ 2 \\ ${ }^{1}$ MIT Kavli Institute for Astrophysics and Space Research, 77 Massachusetts Avenue, 37-582c, Cambridge, MA 02139-4307, USA; aaw @ mit.edu \\ ${ }^{2}$ Department of Astronomy, University of Washington, Box 351580, Seattle, WA 98195, USA \\ ${ }^{3}$ European Southern Observatory, Alonso de Cordova 3107, Casilla 19001, Vitacura, Santiago 19, Chile \\ ${ }^{4}$ Cardiff School of Physics and Astronomy, Cardiff University, Queens Buildings, The Parade, Cardiff, CF24 3AA, UK \\ ${ }^{5} \mathrm{UCO} /$ Lick Observatory, Department of Astronomy and Astrophysics, University of California, Santa Cruz, CA 95064, USA \\ Received 2009 January 31; accepted 2009 June 26; published 2009 July 28
}

\begin{abstract}
We utilize color information for an $\mathrm{H}$ I-selected sample of 195 galaxies to explore the star formation histories and physical conditions that produce the observed colors. We show that the $\mathrm{H}$ I selection creates a significant offset toward bluer colors that can be explained by enhanced recent bursts of star formation. There is also no obvious color bimodality, because the $\mathrm{H}$ I selection restricts the sample to bluer, actively star-forming systems, diminishing the importance of the red sequence. Rising star formation rates are still required to explain the colors of galaxies bluer than $g-r<0.3$. We also demonstrate that the colors of the bluest galaxies in our sample are dominated by emission lines and that stellar population synthesis models alone (without emission lines) are not adequate for reproducing many of the galaxy colors. These emission lines produce large changes in the $r-i$ colors but leave the $g-r$ color largely unchanged. In addition, we find an increase in the dispersion of galaxy colors at low masses that may be the result of a change in the star formation process in low-mass galaxies.
\end{abstract}

Key words: galaxies: evolution - galaxies: fundamental parameters - galaxies: general - galaxies: photometry radio lines: galaxies - surveys

Online-only material: color figures

\section{INTRODUCTION}

The star formation history (SFH), metallicity, and current star formation rate (SFR) all contribute to the observed colors of a galaxy. Remarkably, these factors work in concert to yield a fairly well-defined locus in galaxy color-color space (Strateva et al. 2001). Deviations from this locus as well as the morphology of the locus itself, can lead to significant insight into the underlying processes taking place within galaxies. Many previous studies have utilized the broadband colors of galaxies to investigate the SFH and metallicities of galaxies (e.g., Tinsley 1972; Searle et al. 1973; Tinsley \& Gunn 1976; Balcells \& Peletier 1994; Roberts \& Haynes 1994; de Jong 1996; Bell \& de Jong 2000; Galaz et al. 2002; Gavazzi et al. 2002; Bell et al. 2003; MacArthur et al. 2004; Zackrisson et al. 2005; Driver et al. 2006; Skibba et al. 2008).

The advent of large surveys such as the Sloan Digital Sky Survey (SDSS; York et al. 2000) and the Two Micron All Sky Survey (2MASS; Skrutskie et al. 2006) has created a wealth of uniform data and the statistical foothold to investigate the bimodality of galaxies (Baldry et al. 2004; Kauffmann et al. 2003, 2004), luminosity function (Blanton et al. 2001; Ball et al. 2006), and the average properties of nearby galaxies (Blanton et al. 2003a, 2003b, 2005; Geha et al. 2006; Maller et al. 2009; Skibba et al. 2008). While the large optical and infrared surveys have made large contributions to our understanding of galaxy evolution, they trace only the stellar component of galaxies and do not trace other baryonic material such as cold gas.

Galaxies in the local universe span a range of SFHsfrom blue, gas-rich, low-surface-brightness (LSB) galaxies that are slowly turning their gas into stars with low star-forming efficiencies, to red, gas-poor galaxies that have formed the bulk of their stars in the past. Stars dominate the visible light output of most galaxies, and thus galaxies detected by traditional optical or infrared imaging have well-developed stellar populations. In contrast, the natural way to identify gas-rich, less evolved galaxies is by their $21 \mathrm{~cm} \mathrm{H}$ I radio emission. Aside from its importance for global star formation, a sample of galaxies with both gaseous and stellar information allows for a more complete census of the local baryons (a constraint vital to the calibration of $n$-body simulations; Governato et al. 2007; Brooks et al. 2009).

Previous studies have combined large $\mathrm{H}$ I surveys with optical and infrared samples, namely, the Arecibo Duel Beam and Slice Surveys with the 2MASS (Jarrett et al. 2000; Rosenberg et al. 2005) and the merging of H I Parkes All Sky Survey (HIPASS) with SuperCOSMOS (Hambly et al. 2001a, 2001b, 2001c; Doyle et al. 2005). Rosenberg et al. (2005) were able to probe the baryonic content of a large sample of galaxies, but were limited by the shallow depth of 2MASS, which does not have data for many of the LSB galaxies in the sample. The HIPASS / SuperCOSMOS sample of Doyle et al. (2005) contains optical data for more than $3600 \mathrm{H}$ I-selected galaxies but also suffers from the shallow depth of the SuperCOSMOS optical data.

Recent studies have combined the Parkes H I Equatorial Survey (ES) with the SDSS (Disney et al. 2008; Garcia-Appadoo et al. 2009; West et al. 2009; hereafter W09). The deep SDSS optical data provide information about the stellar content for all $\mathrm{ES} \mathrm{H}$ I sources where the two surveys overlap. In addition, the uniform, accurate, and well-calibrated photometry of the H I-selected ES/SDSS sample allows for a more detailed exploration of the factors affecting galaxy colors, particularly for galaxies with large reservoirs of gas. The optical colors are reasonably sensitive to age, although IR colors are needed to constrain metallicity (Bell et al. 2003). There is thus an unavoidable degeneracy between age and metallicity when using only the optical colors available with SDSS (Bell \& de Jong 
2001; Bell et al. 2003). Our sample does not have a complete set of near-IR counterpart data and some of our results will reflect this limitation.

In this paper, we briefly describe the ES/SDSS sample in Section 2 and examine the colors of $\mathrm{H}$ I-selected galaxies by comparing them to stellar population synthesis models (Section 3.1) and the colors of optically selected galaxies (Section 3.2). We also investigate how line emission affects the broadband colors of gas-rich galaxies (Section 3.3). We demonstrate an increased dispersion in the colors of galaxies at low masses and investigate its possible origins (Section 3.4). We summarize and discuss our results in Section 4.

\section{DATA}

The H I data for our sample come from the Parkes ES (GarciaAppadoo et al. 2009), a blind $\mathrm{H}$ I survey of the southern sky that covers a velocity range from -1280 to $12700 \mathrm{~km} \mathrm{~s}^{-1}$ with an rms noise of $13 \mathrm{mJy}$, using the multibeam receiver on the $64 \mathrm{~m}$ radio telescope in Parkes, Australia. The ES, which is described in detail in an accompanying paper (Garcia-Appadoo et al. 2009), circles the celestial equator between $-6<\delta<+10$ and contains over 1000 sources in $5738 \mathrm{deg}^{2}$. The raw data form part of the HIPASS (Barnes et al. 2001; Meyer et al. 2004; Zwaan et al. 2004; Wong et al. 2006), carried out with the same instrument over the entire sky between $-90<\delta<+25$. However, the ES fields were searched much earlier (Garcia-Appadoo et al. 2009) in readiness for comparison with SDSS data. While the search techniques were much the same as those of the HIPASS team and rely heavily on their procedures, the source lists are not identical. For example, the completeness limit of the ES list is $30 \%$ fainter than the HIPASS limit. This difference is mainly due to our ability to follow up and confirm a higher proportion of the fainter sources, a process that would be impractical with the larger survey. The velocity resolution of the ES H I spectra is $18.0 \mathrm{~km} \mathrm{~s}^{-1}$ and the $3 \sigma \mathrm{H} \mathrm{I}$ mass limit of the survey is $10^{6} \times D_{\mathrm{Mpc}}^{2} M_{\odot}$, assuming a $200 \mathrm{~km} \mathrm{~s}^{-1} \mathrm{H}$ i galaxy profile. For detailed descriptions of the data acquisition, calibration, and reliability, see Garcia-Appadoo et al. (2009) and the HIPASS analysis contained in Barnes et al. (2001), Meyer et al. (2004), and Zwaan et al. (2004).

The optical data for this study come from the SDSS (York et al. 2000; Gunn et al. 1998, 2006; Fukugita et al. 1996; Hogg et al. 2001; Smith et al. 2002; Stoughton et al. 2002; Pier et al. 2003; Ivezić et al. 2004) Data Release 2 (DR2; Abazajian et al. 2004) sky area. The DR2 area is $3324 \mathrm{deg}^{2}$, about half of which overlaps with the equatorial ES region discussed above. Because the SDSS photometric software (РНОTO; Lupton et al. 2002) was not optimized for angularly large galaxies, all of the photometry presented for the ES/SDSS survey has been re-processed using the techniques described in W09. The W09 study describes the optical sample selection and matching to ES and presents the updated SDSS photometry for $195 \mathrm{H} \mathrm{I}-$ selected galaxies using both a sky subtraction procedure that is optimized for angularly large galaxies and a correction for the over-deblending ("shredding") of nearby galaxies by the SDSS pipeline photometry. While more recent SDSS data releases (e.g., DR7; Abazajian et al. 2009) may contain more of the optical counterparts to ES sources, the re-processed photometry is essential for accurate photometric studies and only exists for the DR2 sample (W09).

To be included in the ES/SDSS sample, each candidate galaxy had to meet four criteria: (1) the ES recessional velocity must agree to within twice the velocity width (as measured by

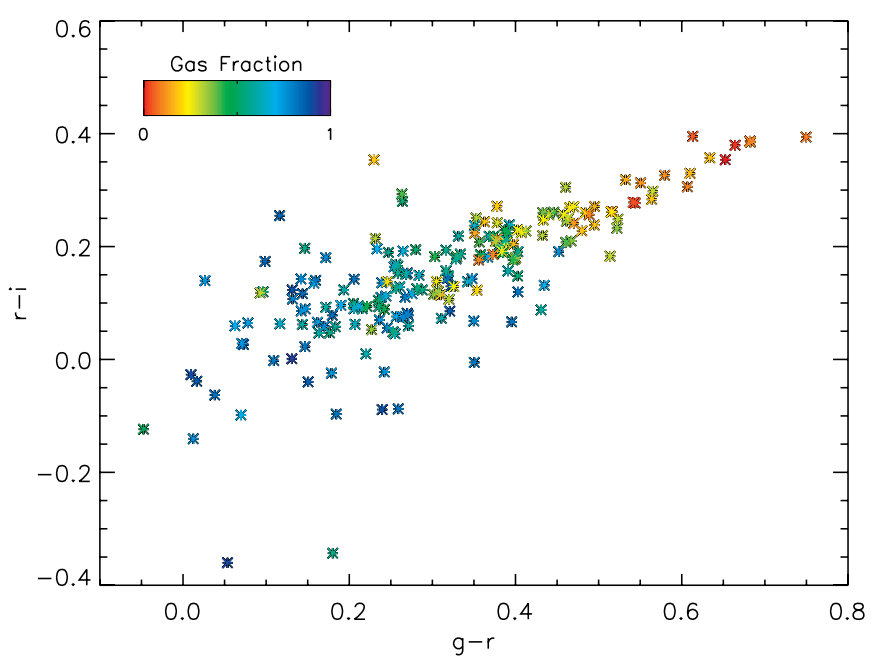

Figure 1. $r-i$ vs. $g-r$ for ES/SDSS sample galaxies. All photometric corrections have been applied to these data. Galaxies have been color coded according to their gas fractions.

(A color version of this figure is available in the online journal.)

the $W_{20}$ value) of the optically derived redshift, (2) there must be no more than one detectable, spatially resolved galaxy within the ES beam at the same redshift, (3) the candidate galaxy must not extend across two or more SDSS fields, and (4) all galaxies must be at least $1^{\prime}$ away from any saturated foreground stars. For additional details on catalog matching, see W09.

\section{RESULTS}

\subsection{Modeling the Colors of H I-Selected Galaxies}

Figure 1 shows the extinction corrected $r-i$ colors of the galaxies in our $\mathrm{H}$ I-selected sample as a function of their $g-r$ color. The galaxies are color coded according to their gas fraction. The gas fraction $\left(f_{\text {gas }}\right)$ is defined as

$$
f_{\text {gas }} \equiv \frac{1.4 M_{\mathrm{HI}}}{1.4 M_{\mathrm{HI}}+M_{\star}},
$$

where $M_{\mathrm{HI}}$ is the $\mathrm{H}_{\mathrm{I}}$ mass and $M_{\star}$ is the stellar mass determined using color-dependent mass-to-light ratios that have been corrected for a Kroupa initial mass function (IMF; Bell et al. 2003; Pizagno et al. 2005; see W09). The factor of 1.4 corrects for the mass in helium. We have neglected the molecular gas component, which is measured to be small in low-mass LSB galaxies that dominate our sample $\left(M_{\mathrm{H}_{2}} / M_{\mathrm{H}_{\mathrm{I}}}<0.1\right.$; Schombert et al. 1990; Matthews et al. 2005). No molecular gas measurements (CO) exist for any of our sample galaxies. However, recent $\mathrm{CO}$ observations of nearby galaxies give some insight into the range of molecular gas content in galaxies of similar morphological type. We estimate that the molecular to neutral gas fraction $\left(M_{\mathrm{H}_{2}} / M_{\mathrm{H}_{\mathrm{I}}}\right)$ may be as high at 0.5 for some of the early-type spirals in the ES/SDSS sample (Leroy et al. 2009). Thus, we may be underestimating the gas fractions in a small number of our galaxies.

Figure 1 shows several important trends. First, the H I-selected galaxies occupy a well-defined locus in color, as seen in other SDSS studies (Strateva et al. 2001; Blanton et al. 2003a; Baldry et al. 2004; Maller et al. 2009). Second, the gas fraction scales with galaxy color such that bluer galaxies have a larger fraction of their baryonic mass in gas, as has also been reported for less uniform samples (Kannappan 2004). Third, the scatter in color-color space is large for gas-rich galaxies. 
To interpret the trends observed in Figure 1, we compared the colors of the ES/SDSS sample galaxies to theoretical predictions by running a series of Bruzual \& Charlot (2003) population synthesis models at various metallicities and SFHs. The models use the Chabrier (2003) IMF to model the stellar populations with a range of stellar masses of $0.6 M_{\odot} \leqslant m \leqslant$ $120 M_{\odot}$, at six different metallicities $(0.005,0.02,0.2,0.4$, 1 , and 2.5 times solar) and 10 different SFHs. The SFHs are modeled as having either (1) an exponentially decreasing SFR $\left(e^{-t / \tau}\right)$ with $\tau$ values of $8,4,2$, and $1 \mathrm{Gyr}$ (these models have mean, mass-weighted stellar ages of 7.4, 8.6, 10.0, and $11.0 \mathrm{Gyr}$, respectively); (2) an exponentially increasing SFR with $\tau$ values of $8,4,2$, and $1 \mathrm{Gyr}$ (mean stellar ages of 4.6, 3.4, 2.0, and $1.0 \mathrm{Gyr}$, respectively); (3) a continuous $\operatorname{SFR}(\tau=\infty$; mean stellar age of $6 \mathrm{Gyr}$ ); or (4) an instantaneous event of star formation ( $\tau=0$; mean stellar age of $12 \mathrm{Gyr}$ ). For each model, we assume that 12 Gyr has elapsed since the first formation of stars (Dalcanton \& Bernstein 2002; Brooks et al. 2009) and extract the SDSS model colors at that epoch. We do not include dust in any of the population models because we have corrected for the internal extinction of the galaxies using the measured rotation velocities (H I line widths) and the prescription of Tully et al. (1998), where galaxies with the same rotation velocity are assumed to have similar internal dust extinction. The uncertainty in this correction is $\sim 0.1 \mathrm{mag}$ and may increase the scatter of our photometry. However, many of the galaxies in the SDSS / ES sample have little or no dust correction due to their slow rotation (Tully et al. 1998; Dalcanton et al. 2004; see W09 for more details).

Each Bruzual \& Charlot (2003) model was run without gas recycling - the population of stars maintains the same metallicity throughout the SFH. While this does not accurately mimic the chemical evolution of individual galaxies (which are some amalgamation of ages and metallicities), it does provide a set of specific model comparisons by which to compare the mean properties of observed systems.

Figure 2 shows the resulting population grid for the $r-i$ colors as a function of $g-r$. The "horizontal" lines are lines of constant metallicity and the "vertical" lines are lines of constant $\tau$ (a proxy of mean stellar age). Tracks of constant age are well separated in $g-r$, making $g-r$ a good probe of stellar age. On the other hand, the $r-i$ color is only marginally sensitive to metallicity and the aforementioned degeneracy is apparent at all subsolar metallicities. These grids bracket all reasonable continuous SFHs, but bracket only a narrow region of $g-r$ versus $r-i$ color space. Although SDSS provides photometry in five different photometric passbands, most of our color analysis uses only the $g, r$, and $i$ bands. For most of our galaxies, the $u$ and $z$ bands yield photometry with large uncertainties that does not help us differentiate between models or constrain photometric relationships. Because the $u$ band is sensitive to the SFR (Hopkins et al. 2003), we have included $u$-band photometry in a limited amount of our analysis.

The ES/SDSS sample galaxies have been overplotted and color coded according to their gas fractions, as in Figure 1. At the red end of the galaxy distribution, the colors are well explained by the model grids. The redder galaxies are consistent with having SFHs that have been declining to the present. These results are in agreement with previous work by Bell et al. (2003) as well as Juneau et al. (2005), who demonstrate that galaxies with large stellar masses formed most of their star in the first $3 \mathrm{Gyr}$ after their formation. The reddest galaxies also have colors indicative of super-solar metallicities. These red $(g-r>0.6)$,

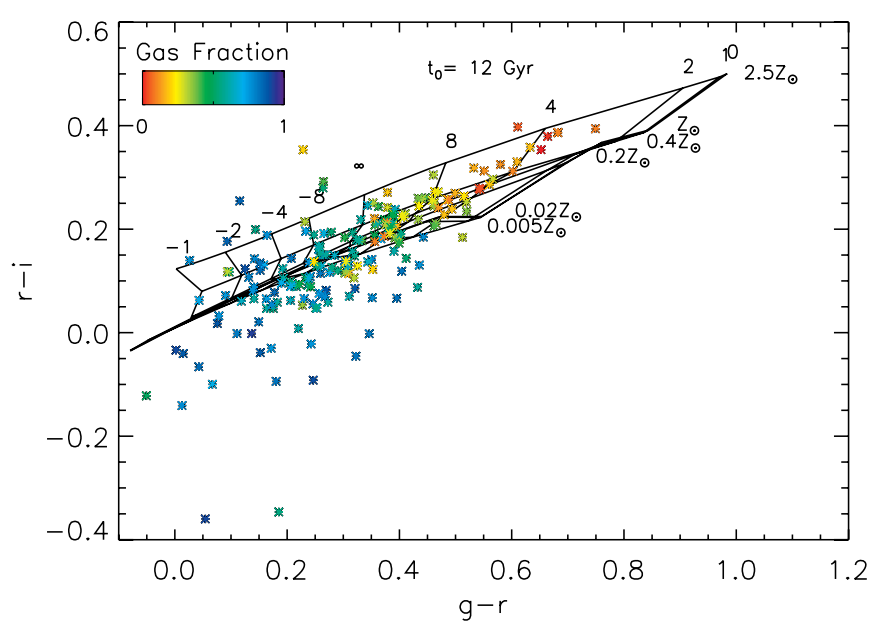

Figure 2. $r-i$ vs. $g-r$ for $\mathrm{H}$ I-selected galaxies. Galaxies have been color coded according to their gas fractions. Bruzual \& Charlot (2003) population synthesis grids have been overplotted for various continuous SFH and metallicities. The SFHs are given by $\tau$ values for an exponential SFR starting at $12 \mathrm{Gyr}$ ago. $\left(e^{-t / \tau}\right)$. Metallicities are plotted in comparison to solar.

(A color version of this figure is available in the online journal.)

high-metallicity galaxies are all early-type spiral, lenticular, or elliptical galaxies.

The data and models in Figure 2 suggest that the red galaxies formed almost all of their stars in the distant past and have been undergoing little to no star formation since, in spite of containing $\mathrm{H} \mathrm{I}$ at the present. Some mechanism is therefore responsible for the continued presence of $\mathrm{H}$ I. The cannibalization of gas-rich dwarfs or infall from "cold mode" accretion (Katz et al. 2003; Brooks et al. 2009) might be sufficient to explain the current small reservoir of gas, which is only a small fraction of the galaxies' baryonic mass. Regardless of the physical process by which these galaxies acquired or maintained gas, the presence of $\mathrm{H}$ I suggests that recent star formation is likely to have taken place. Indeed, all of the galaxies redder than $g-r>$ 0.5 have SDSS spectra that contain emission lines. In spite of the aperture bias in SDSS fiber spectroscopy (fibers are only $3^{\prime \prime}$ in diameter), these spectra can be useful for determining gross spectroscopic properties. Based on their location on a BaldwinPhillips-Terlevich diagram (Baldwin et al. 1981), five of the 156 emission-line galaxies are consistent with hosting an active galactic nucleus (AGN). All five of the galaxies with AGNs have PetroR90 radii (radii containing 90\% of the Petrosian flux) larger than $30^{\prime \prime}$. Therefore, their disk emission (not the AGN) dominates the integrated photometry and they are included in the remainder of the analysis. The majority of the galaxies in the ES/SDSS sample have emission lines that suggest that current star formation is underway in these galaxies.

As we look to the bluer galaxies in Figure 2, the inferred metallicities are lower and the SFHs move toward a constant SFR $(\tau=\infty)$. This shift is likely due to a decrease in star formation efficiency. We define star formation efficiency as the efficiency in converting gas into stars. As discussed above, we neglect the role of molecular gas (the state of gas from which stars form), which should be irrelevant for the bluer galaxies in our sample. Recent studies (that included the molecular component) have seen a similar decrease in star formation efficiency in nearby, H I-dominated galaxies (Bigiel et al. 2008). Although we do not resolve the $\mathrm{H}$ I emission, the failure to convert large amounts of $\mathrm{H}$ I into stars in the blue galaxies constitutes a decrease in star-forming efficiency. Such a decrease 


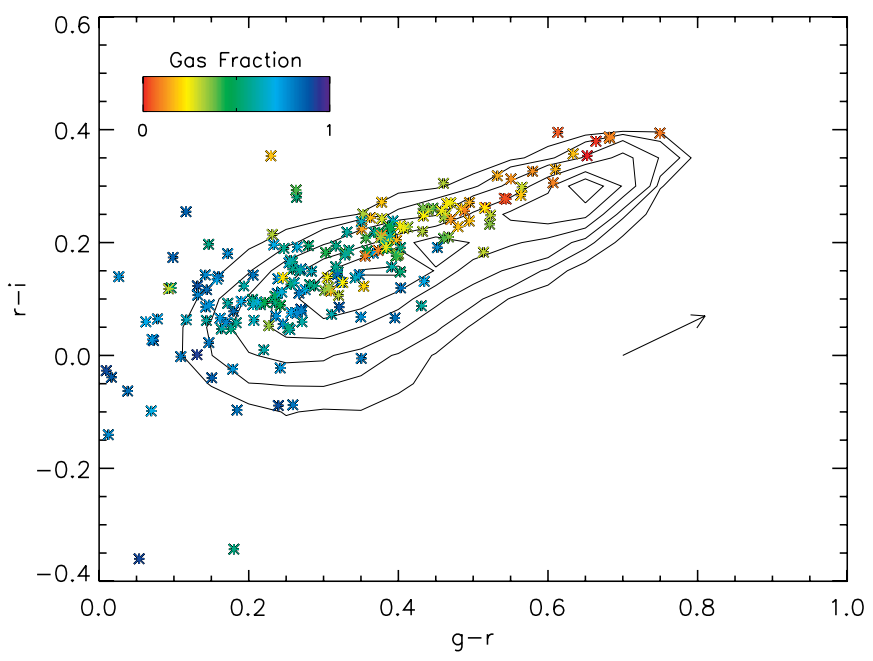

Figure 3. $r-i$ vs. $g-r$ for $\mathrm{H}$ I-selected galaxies (colored symbols) plotted with a volume-selected sample of galaxies from SDSS (contours). No internal extinction has been applied to these data but a reddening vector has been plotted for reference. Galaxies in the thesis sample have been color coded according to their gas fractions. Note that the $\mathrm{H}$ I-selected sample appears to have colors that are shifted off of the SDSS galaxy locus.

(A color version of this figure is available in the online journal.)

slows the rate of star formation, keeping metallicities low and gas fractions high, allowing stars to form at the present day.

The bluest galaxies $(g-r<0.3)$ are best modeled by SFHs that have increasing SFRs. This area of the model parameter space has been ignored by previous galaxy studies (Kauffmann et al. 2003; Brinchmann et al. 2004; Salim et al. 2005; Johnson et al. 2007). Gas infall might serve to increase the H I surface density and subsequently the SFR in these galaxies. Although recent bursts have likely occurred in these galaxies, the already blue $g-r$ colors of these do not change significantly when recent bursts are added to the models (see Section 3.3).

Many of the bluest galaxies in the ES/SDSS sample have colors that are not consistent with any of the Bruzual and Charlot models. Most of these galaxies have $r-i$ colors that are too blue to be explained by any iteration of the models. The LSB nature of many of these systems suggests that for even small SFR, emission lines might be able to dominate the broadband colors (Zackrisson et al. 2001, 2005, 2008; Magris et al. 2003; Anders \& Fritze-v. Alvensleben 2003). We investigate this possibility in detail in Section 3.3.

Finally, there are a five galaxies that seem to be too red in $r-i$ to be explained by any population models. Two of these galaxies have a saturated star nearby that is most likely affecting their colors. One of the galaxies has very large photometric uncertainties because of its LSB and the other two have very compact cores, which suggests the presence of AGNs. If we compute the colors for these two galaxies excluding the central region, using a shell with radial boundaries defined to be from half of the Petrosian radius to the Petrosian radius, both galaxies fall back to or below the model grids when their central core is excluded.

\subsection{The Colors of H I Versus Optically Selected Galaxies}

Although the trends seen in Figure 1 have been noted in optically selected SDSS samples (e.g., Strateva et al. 2001; Blanton et al. 2003a), the H I selection introduces a systematic shift in the color distribution of the ES/SDSS sample galaxies. Figure 3 shows the $r-i$ versus $g-r$ colors for the ES/SDSS

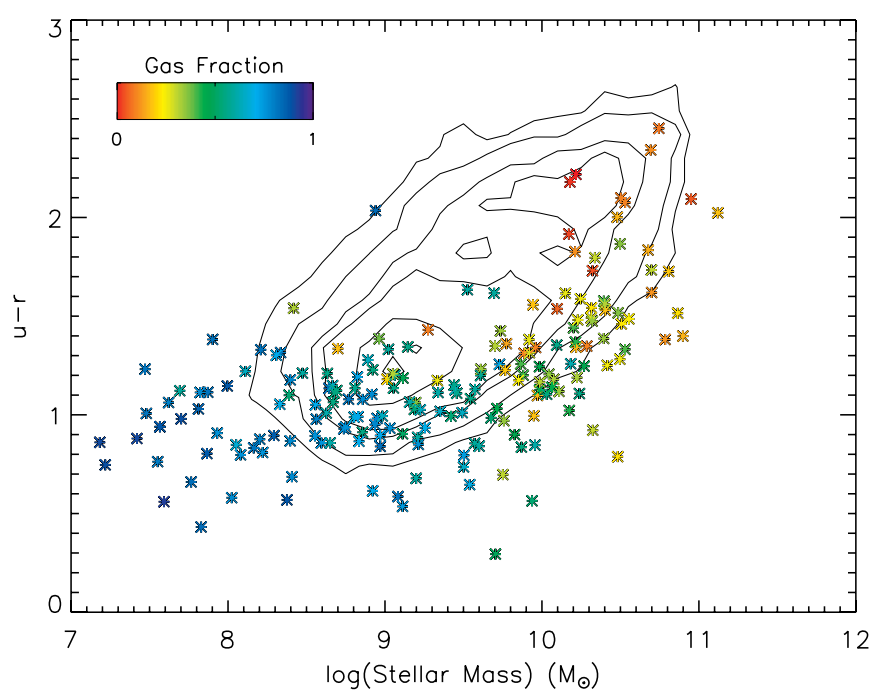

Figure 4. $u-r$ colors as a function of stellar mass for the ES/SDSS galaxies (colored according to gas fraction) and the SDSS DR4 sample (contours). The bimodal galaxy distribution (blue and red sequence) is easily seen in the SDSS DR4 galaxies, but absent from the ES/SDSS galaxies. The $\mathrm{H}$ I selection of the ES/SDSS galaxies identifies gas-rich galaxies that are on the blue sequence and are currently in the process of star formation.

(A color version of this figure is available in the online journal.)

sample plotted on top of a volume-limited sample drawn from the SDSS DR4 sample (Adelman-McCarthy et al. 2006). All galaxies have been $k$-corrected and corrected for Milky Way extinction. We used the Milky Way extinction values from Schlegel et al. (1998) and calculated $k$-corrections using the IDL package kcorrect_v3.2 (Blanton et al. 2003b). The Blanton et al. (2003b) $k$-correction method uses empirically derived eigenspectra to generate model spectra from SDSS photometry. All of the galaxies (both SDSS DR4 and ES/SDSS) were corrected using the same method to $z=0$. Most of the $k$-corrections were smaller than $0.01 \mathrm{mag}$ in all bands. No data in Figure 3 have been corrected for internal extinction. The reddening vector (derived from Schlegel et al. 1998) has been included to show that any correction to either data set is almost perfectly aligned with the galaxy locus.

The $\mathrm{H}$ I-selected galaxies do not show the color bimodality seen in previous SDSS studies (e.g., Blanton et al. 2003a; Baldry et al. 2004). Figure 4 shows the $u-r$ colors as a function of stellar mass for the ES/SDSS galaxies (colored according to gas fraction) and the SDSS DR4 sample (contours). The bimodal galaxy distribution (blue and red sequence) is easily seen in the SDSS DR4 galaxies, but is absent from the ES/SDSS galaxies. The H I selection of the ES/SDSS galaxies identifies gas-rich galaxies that are on the blue sequence and are currently in the process of star formation. Like in Figure 3, the ES/SDSS galaxies in Figure 4 appear to be offset from the SDSS DR4 sample (bluer in $g-r$ or redder in $r-i$ ). Figure 4 also demonstrates the ability of an $\mathrm{H}$ I-selected sample to recover galaxies with small stellar masses; the ES/SDSS sample has a significant number of galaxies with stellar masses below $10^{8} M_{\odot}$.

It is clear from Figure 3 that the $\mathrm{H}$ I-selected sources fall to one side of the SDSS galaxy distribution, such that they are either bluer in $g-r$ or redder in $r-i$. This offset is particularly pronounced at the red end of the color distribution where the color dispersion is small. As noted in the previous section, the redder galaxies have SFHs that suggest that almost all star 


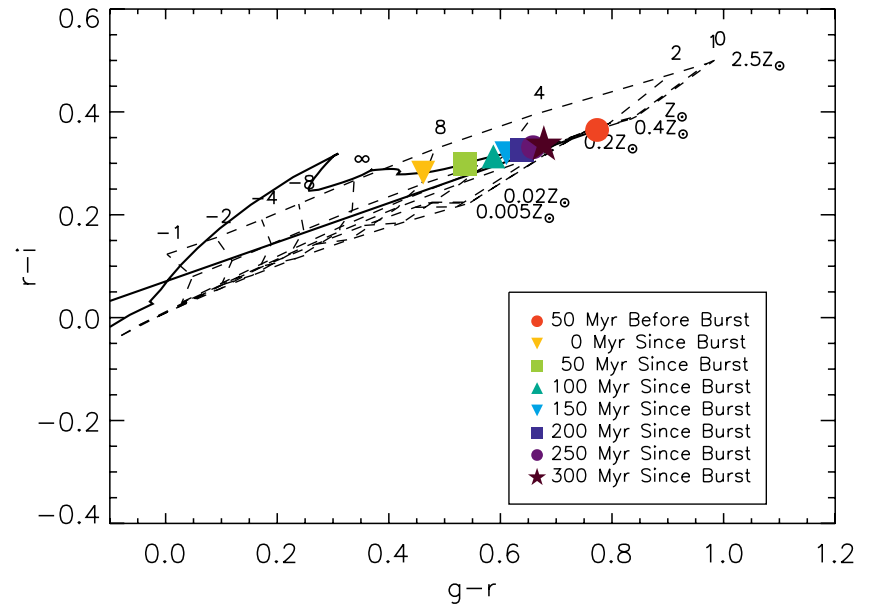

Figure 5. $r-i$ vs. $g-r$ evolution of a single Bruzual and Charlot model with the addition of an instantaneous burst of star formation that produced $1 \%$ of the total stellar mass of the galaxy and occurred $300 \mathrm{Myr}$ in the past (solid line). The Bruzual and Charlot continuous star formation grid (from Figure 2) is plotted for reference (dashed lines). The continuous model to which the burst was added corresponds to a red galaxy from the ES/SDSS sample ( $\tau=2$, $Z=Z_{\odot} ; g-r$ and $r-i$ colors of 0.75 and 0.3 , respectively). Colored symbols are plotted every $50 \mathrm{Myr}$ starting $50 \mathrm{Myr}$ before the burst. Colors bluer than $g-r<0.4$ and $r-i<0.2$ are observed only for very short durations ( $<1 \mathrm{Myr}$ ). By $100 \mathrm{Myr}$, the colors have reddened to within $\Delta g-r=0.15$ and $\Delta r-i=$ 0.05 of their initial value and continue to redden for hundreds of Myr.

(A color version of this figure is available in the online journal.)

formation occurred in the distant past. The presence of $\mathrm{H} \mathrm{I}$ in these systems may indicate a recent acquisition of $\mathrm{H}$ I and a subsequent ignition of star formation, as confirmed by their SDSS spectra. This temporary increase in SFR due to latetime accretion would naturally explain why the red $\mathrm{H}$ I-selected galaxies are slightly bluer in $g-r$ than the rest of the SDSS galaxy population (Figure 3 ).

To constrain the amount of recent star formation needed to produce the observed color offset between the ES/SDSS galaxies and the "main" SDSS sample, we re-ran the same series of Bruzual \& Charlot (2003) population synthesis codes as in Figure 2, but with the addition of a recent burst of star formation. We generated an initial model with $g-r$ and $r-i$ colors of 0.75 and 0.3 , respectively $\left(\tau=2 ; Z=Z_{\odot}\right)$. We then added a single instantaneous burst (delta peak) of star formation to the continuous model and varied the time of the burst from 2 Gyr to $100 \mathrm{Myr}$ in the past. We also varied the burst strength from $0.1 \%$ to $10 \%$ of the integrated star formation. The resulting models were most sensitive to the time at which the burst was placed. The models that most closely matched the slight $g-r$ bluing were bursts placed at $300 \mathrm{Myr}$ in the past with $1 \%$ of the past integrated star formation occurring in the burst.

Figure 5 shows the evolutionary track for the best-matched model burst (300 Myr in the past) with symbols plotted every 50 Myr since burst. The figure demonstrates that there is a rapid and short-lived change in color that occurs in the first few Myr and is followed by a slow reddening for hundreds of Myr. Colors bluer than $g-r<0.4$ and $r-i<0.2$ are observed only for very short durations ( $<1 \mathrm{Myr}$ ). By $100 \mathrm{Myr}$ the colors have reddened to within $\Delta g-r=0.15$ and $\Delta r-i=0.05$ of their initial value and continue to redden for hundreds of Myr. This "bottleneck" in the burst evolution suggests that the small color offset observed in Figure 3 could be sufficiently long-lived to be due to a burst in the past few hundred Myr.

Figure 6 shows the ES/SDSS galaxies and the model grids for continuous star formation with an additional $1 \%$ burst occurring

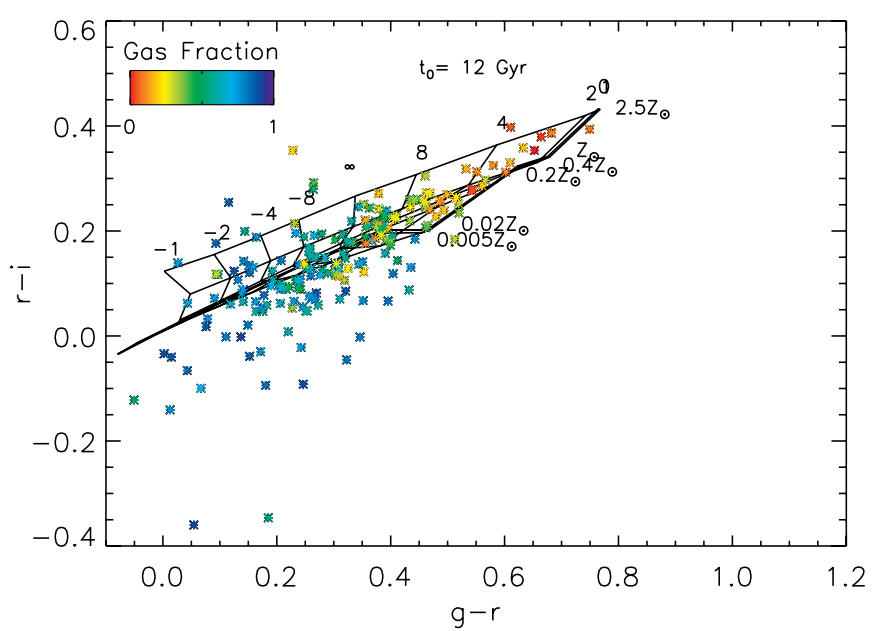

Figure 6. $r-i$ vs. $g-r$ for $\mathrm{H}$ I-selected galaxies. All photometric corrections have been applied to these data. Galaxies have been color coded according to their gas fractions. Bruzual \& Charlot (2003) population synthesis grids have been overplotted for various SFH and metallicities after 12 Gyr of star formation. The SFH are given by $\tau$ values for an exponential $\operatorname{SFR}\left(e^{-t / \tau}\right)$. These grids include an instantaneous burst of star formation that produced $1 \%$ of the stellar mass, $300 \mathrm{Myr}$ in the past.

(A color version of this figure is available in the online journal.)

$300 \mathrm{Myr}$ in the past. The red $\mathrm{H}$ I-selected galaxies in the burst model have $\tau \sim 3$, compared to the $\tau \sim 4$ seen in the burstfree models. By comparing the difference between the grids in Figures 2 and 6 with the offset observed in Figure 3, it is clear that the $\mathrm{H}$ I-selected galaxies in the burst case (Figure 6) occupy the same model space $(\tau \sim 3)$ as the SDSS "main" galaxies in the burst-free case (Figure 2). Unfortunately, our inability to correct for internal extinction in the SDSS "main" sample prohibits us from overlaying accurate grids on Figure 3, which would be highly instructive. However, "blinking" back and forth between Figures 2 and 6 confirms that a recent burst can explain the offset of the red galaxies.

It is important to note that the addition of the burst may effect the colors of the blue galaxies as well. A single instantaneous burst produces extremely blue colors for very short times. However, in reality, the bursts are not instantaneous, but instead last for a finite amount of time with some exponential decrease (10-300 Myr depending on galaxy type; McQuinn et al. 2009). If a significant fraction of the star formation has occurred in the very recent past, then some of the blue colors may be due to recent bursts. However, rising SFRs are still required to explain the bulk of the galaxies with colors bluer than $g-r<0.3$.

Although degeneracies between the time, duration, and strength of the star formation events in the models prevent an exact characterization of the bursts in the $\mathrm{H}$ I-selected galaxies, we can safely claim that a new generation of stars has been formed in the last few hundred Myr in the red systems (a large instantaneous burst has a similar effect on the integrated colors as a small exponentially decreasing burst). The bursts require no more than $1 \%$ of the mass to be involved as long as the burst was in the last 300 Myr.

We note that the bluer $g-r$ colors in the red ES/SDSS galaxies may not be solely due to main-sequence stars, since most blue main-sequence stars will have ceased their hydrogen burning after a few hundred Myr; the $g-r$ color of a mainsequence turnoff star at $300 \mathrm{Myr}$ is $\sim 0.0$ (Sarajedini et al. 2004; Covey et al. 2007). We suggest that another possibility for the blue $g-r$ colors we see in the red $\mathrm{H}$ I-selected galaxies could 

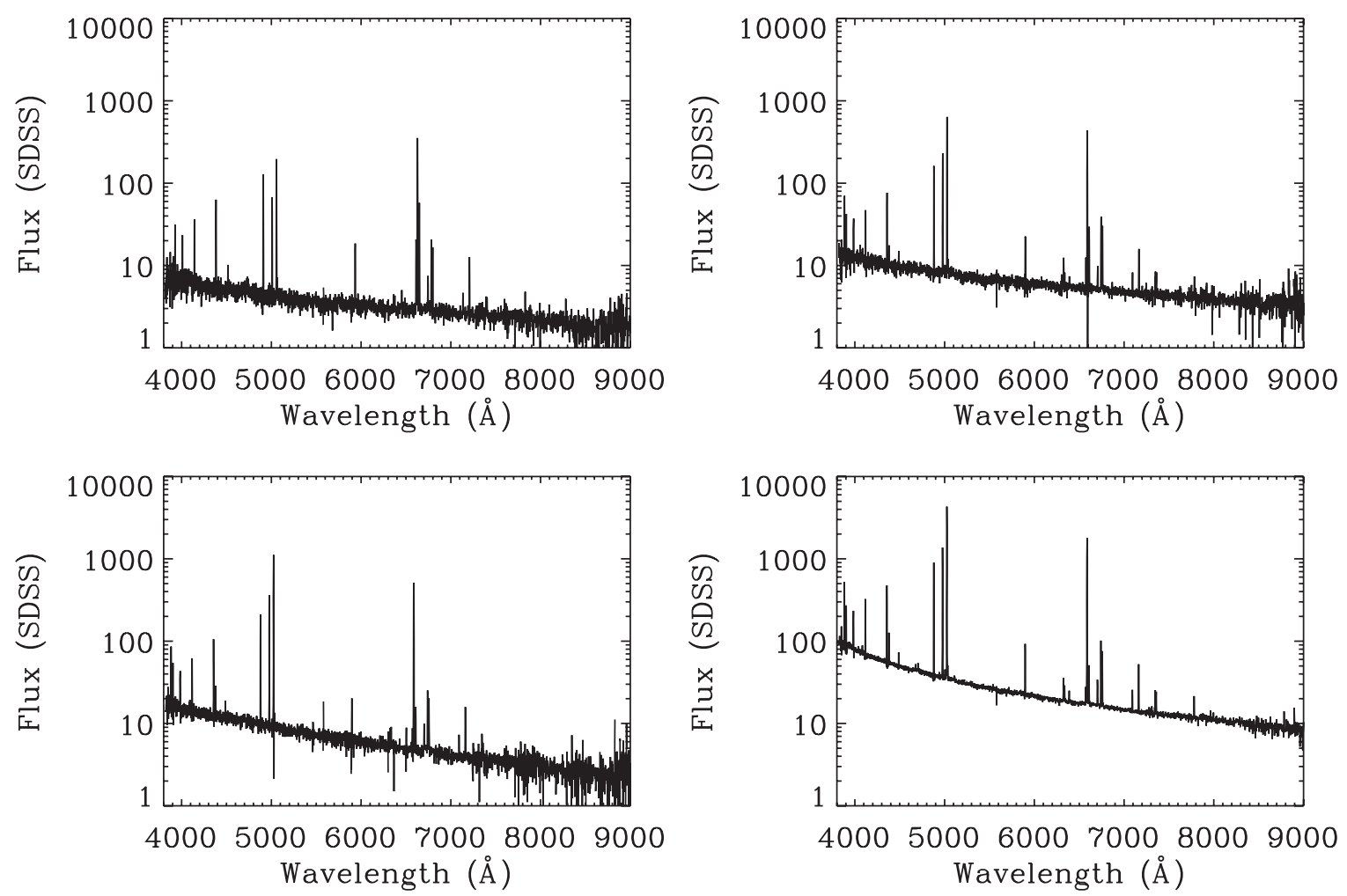

Figure 7. SDSS fiber spectra for four galaxies in the ES/SDSS sample with strong emission-line features. All of the fibers for these spectra were placed on top of $\mathrm{H}$ II regions in the galaxies. The flux axis is in units of $10^{-17} \mathrm{erg} \mathrm{s}^{-1} \mathrm{~cm}^{-2} \AA$.

be due to an elevated level of BHeB stars left over from a recent burst of star formation. These stars are seen several hundred Myr after a star formation event (Dohm-Palmer et al. 2002).

\subsection{Modeling Galaxy Colors with Emission Lines}

Even with the addition of bursts, the colors of many ES/SDSS galaxies do not fall on the Bruzual \& Charlot (2003) model grids. Unlike the red galaxies, which can be easily explained, the majority of the discrepant galaxies are significantly bluer in $r-i$ than any models can predict. These systems also have high gas fractions and most have LSB. No matter what parameters we alter in the Bruzual and Charlot models, we cannot produce a stellar population with very blue $r-i$ colors.

We show that the discrepant colors can be explained by including the emission lines that dominate the spectra of these outlying galaxies. The blue colors and high gas fractions of the discrepant galaxies suggest that they are actively forming stars. Because these galaxies also have LSBs, it is possible that emission lines produced by $\mathrm{H}$ II regions contribute significantly to their luminosity. $\mathrm{H}$ II regions produce numerous emission lines in the $g$ and the $r$ bands. $\mathrm{H} \alpha$ dominates in the $r$ band while [O III] and $\mathrm{H} \beta$ dominate in the $g$ band. In contrast, the $i$ band has almost no emission lines. Therefore, in an $\mathrm{H}$ II region, we would expect the $r-i$ color to be significantly bluer than the underlying stellar continuum and the $g-r$ color to change only slightly. This suggests that $i$-band luminosity may be a better indicator of the underlying stellar mass than $g-r$. It also implies that the $g-r$ color should be used to compute color-dependent mass-to-luminosity ratios (the emission lines tend to cancel out; see below).

We can test this idea using the SDSS spectroscopic fibers, which were often placed on $\mathrm{H}$ II regions for LSB galaxies. By comparing the colors of $\mathrm{H}$ II regions (derived from SDSS spectra) with the integrated colors of the galaxies that host them, we can quantify color changes produced by $\mathrm{H}$ II regions.

We first selected 10 ES/SDSS galaxies with SDSS spectroscopy and required that they have large $\mathrm{H} \alpha$ equivalent widths ( $>300 \AA$ ). Four of the spectra selected are shown in Figure 7. All of the selected spectra have good signal to noise $(>50)$ and are dominated by emission-line features. We visually inspected each galaxy to ensure that all of the spectra selected (and shown in Figure 7) are from fibers placed on high-surface brightness $\mathrm{H}$ II regions. We convolved the $g, r$, and $i$ SDSS filter curves with each spectrum and converted the computed flux density to $\mathrm{AB}$ magnitudes using the relation that a magnitude 0 object has a flux density of $3631 \mathrm{Jy}$ (Oke \& Gunn 1983). We then compared the integrated colors of the 10 galaxies derived in W09 with the $\mathrm{H}$ II region colors derived from the spectra.

Figure 8 shows the resulting differences between the $\mathrm{H}$ II region and the integrated color of each galaxy. The colors derived from fibers placed on $\mathrm{H}$ II regions are plotted as black symbols and the integrated galaxy colors derived from images (W09) are plotted as red diamonds. Blue lines connect a galaxy's integrated color to the color of the $3^{\prime \prime}$ diameter aperture around the $\mathrm{H}$ II regions. The $\mathrm{H}$ II region colors are bluer in $r-i$ by $\sim 0.5^{m}$ and all but two become bluer in $g-r$ by $\sim 0.2^{m}$, compared to the integrated colors. This result confirms the hypothesis that emission lines can make the $r-i$ color blue enough to explain the colors we see in the ES/SDSS sample. However, contrary to our initial expectation, the $g-r$ color does change considerably. The bluer $g-r$ colors near $\mathrm{H}$ II regions can be explained as being produced by a young underlying stellar population. More surprisingly, the colors near two of $\mathrm{H}$ II regions are redder in $g-r$ than the integrated light from the galaxies. This difference may reflect the presence of dust localized to the $\mathrm{H}$ II region.

To further test the emission-line hypothesis as well as to confirm the reason for the $g-r$ color change in Figure 8, 


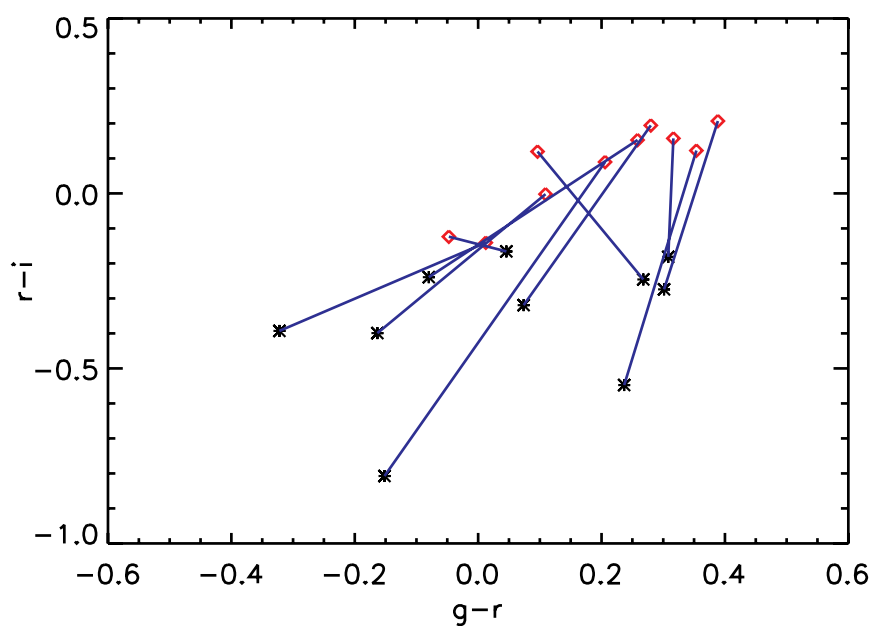

Figure 8. $r-i$ vs. $g-r$ color difference between the integrated colors of 10 galaxies (red diamonds) and the "fiber" magnitudes computed from the H II region spectra (black symbols). The blue lines connect each galaxy's integrated color to its $\mathrm{H}$ II region color. All of the galaxies get bluer in $r-i$ and all but two get bluer in $g-r$.

(A color version of this figure is available in the online journal.)

we added model emission lines to the computed Bruzual and Charlot model colors. Theoretical models for line emission in galaxies are given by Kewley et al. (2001) using the STARBURST99-MAPPINGS III code. The models cover a range of metallicities, ionization states, electron densities, and assumption about the burst lengths. For each model, the wavelength of every line and its energy are provided. We convolved these energies with the SDSS filters and converted the computed flux densities into AB magnitudes. We added the emission-line output to the Bruzual and Charlot colors using a scaling coefficient that determines the strength of the emission line relative to the true stellar continuum. This coefficient is directly proportional to the SFR and can be used to estimate the range of possible SFRs for a galaxy. The final models are not fully selfconsistent, because the ionizing radiation for the emission-line spectrum does not come directly from the Bruzual and Charlot spectrum. However, because the star formation is highly localized in late-type galaxies, it is possible for the ionizing radiation in isolated $\mathrm{H}$ II regions to be different than that of the broader component of stars

When adding the STARBURST99-MAPPINGS III emissionline models to the Bruzual and Charlot outputs, we ensured that the adopted gas metallicity matched the assumed stellar metallicity. Because there are only three overlapping metallicities, we limited our analysis to $0.2 Z_{\odot}, 0.4 Z_{\odot}$, and solar metallicity populations. In reality most of the galaxies where emission lines dominate are low mass and gas rich and thus are unlikely to have high metallicities. Even values of $0.2 Z_{\odot}$ might be too high for these systems (Tremonti et al. 2004).

Figures 9 and 10 show the colors $(r-i$ versus $g-r$ and $u-r$ versus $g-r$, respectively) that result from adding emission lines to a single Bruzual and Charlot model ( $\tau=8 \mathrm{Gyr} ; Z=0.4 Z_{\odot}$ ). This particular model was chosen because it lies on the red end of the blue population and intersects the ES/SDSS color-color locus. The colored symbols represent different SFRs. In Figure 9, the model galaxy's $r-i$ color becomes bluer as star formation is increased, while the $g-r$ color is almost unchanged. A similar effect is seen in the $u-r$ color in Figure 10. This confirms that the non-zero $g-r$ color offsets near H II regions must indeed be due to stellar populations and or dust.

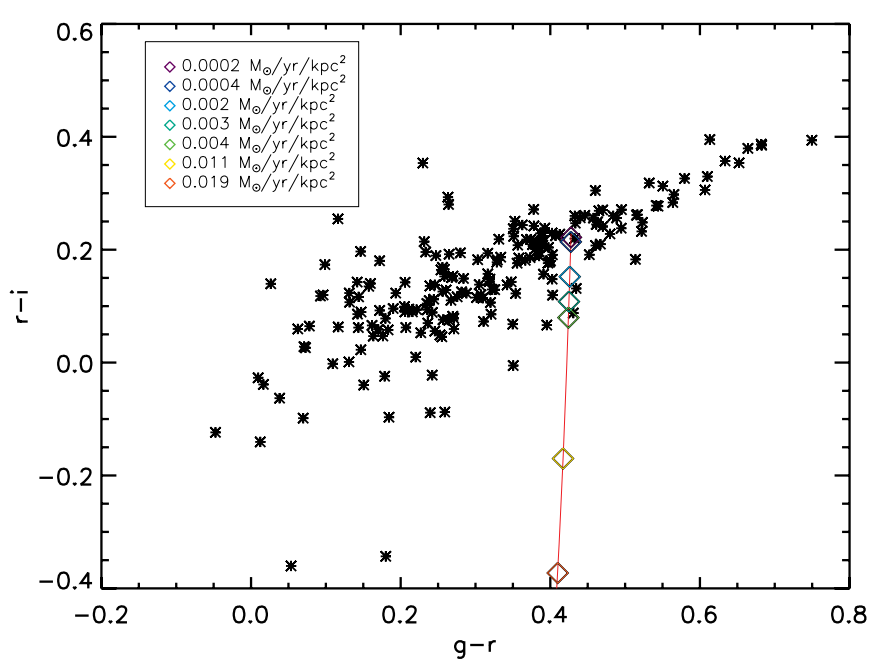

Figure 9. $r-i$ vs. $g-r$ colors of the ES/SDSS galaxies with the addition of emission lines to an underlying stellar population for a single Bruzual and Charlot model galaxy $\left(\tau=8 \mathrm{Gyr} ; Z=0.4 Z_{\odot}\right.$ ). The colored symbols represent different star formation rates. The galaxy's $r-i$ color becomes bluer as star formation is increased, while the $g-r$ color goes almost unchanged.

(A color version of this figure is available in the online journal.)

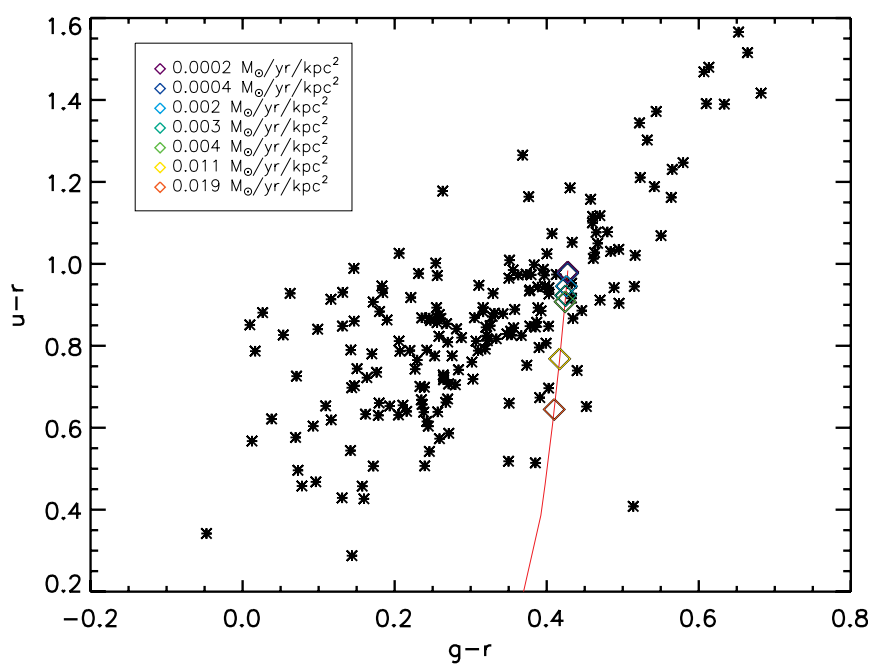

Figure 10. $u-r$ vs. $g-r$ colors of the ES/SDSS galaxies with the addition of emission lines to an underlying stellar population for a single Bruzual and Charlot model galaxy $\left(\tau=8 \mathrm{Gyr} ; Z=0.4 Z_{\odot}\right.$ ). The colored symbols represent different star formation rates.

(A color version of this figure is available in the online journal.)

In Figure 11, we show stellar population grids similar to those in Figure 2, but with an 8 Myr long continuous burst of star formation. Black lines indicate emission-line models with an electron density of $10 \mathrm{~cm}^{-3}$ and an ionization parameter of $5 \times 10^{6} \mathrm{~cm} \mathrm{~s}^{-1}$. Red lines have the same electron density but an ionization parameter of $8 \times 10^{7} \mathrm{~cm} \mathrm{~s}^{-1}$. This plot demonstrates that adding emission lines of varying strength can explain all of the discrepant blue galaxies. While $8 \mathrm{Myr}$ may be short for some realistic starburst events, it successfully demonstrates the effect of current (or recent) star formation on the colors of galaxies.

We found that varying the electron density and burst length (8 Myr continuous versus instantaneous) did not have a major effect on the output models. All significant changes in color are driven by the ionization parameter and by the global normalization of the emission-line spectrum, which is directly proportional to the SFR (the emission lines are generated for 


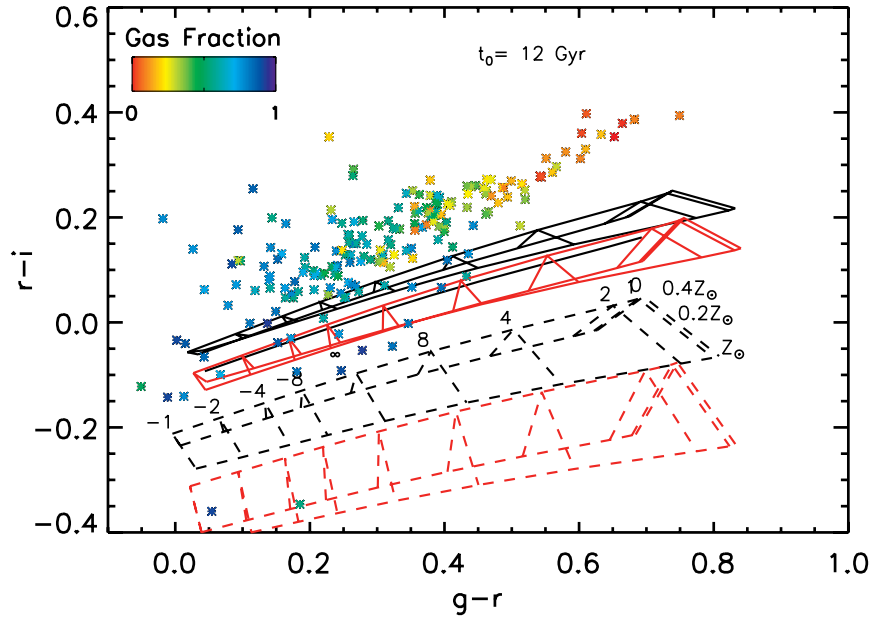

Figure 11. $r-i$ vs. $g-r$ colors for the $\mathrm{H}$ I-selected galaxies. Color coding indicates the gas fractions of the galaxies. The grids represent two different linear combinations of the STARBURST99-MAPPINGS III emission-line models and the Bruzual and Charlot population synthesis models. The Bruzual and Charlot models are identical to those shown in Figure 2. All emission-line models use an $8 \mathrm{Myr}$ continuous burst of star formation. Black lines indicate emission-line models with an electron density of $10 \mathrm{~cm}^{-3}$ and an ionization parameter of $5 \times 10^{6} \mathrm{~cm} \mathrm{~s}^{-1}$. Red lines have the same electron density but an ionization parameter of $8 \times 10^{7} \mathrm{~cm} \mathrm{~s}^{-1}$. Solid lines have SFR densities of $1.2 \times 10^{-3} M_{\odot}$ $\mathrm{yr}^{-1} \mathrm{kpc}^{-2}$ and $3.7 \times 10^{-3} M_{\odot} \mathrm{yr}^{-1} \mathrm{kpc}^{-2}$ for the two models (black and red), respectively. The dashed lines have SFRs three times larger than the solid lines. These models clearly describe the colors of all of the galaxies that are not fitted by normal populations of stars.

(A color version of this figure is available in the online journal.)

a specific value of the SFR). Solid lines in Figure 11 have SFR densities of $1.2 \times 10^{-3} M_{\odot} \mathrm{yr}^{-1} \mathrm{kpc}^{-2}$ and $3.7 \times 10^{-3}$ $M_{\odot} \mathrm{yr}^{-1} \mathrm{kpc}^{-2}$ for the two models (black and red), respectively. The dashed lines have SFRs three times larger than the solid lines. For typical galaxies in our sample, we estimate SFRs of 0.004-0.2 $M_{\odot} \mathrm{yr}^{-1}$ in the emission-line-dominated regions. These are reasonable SFRs for small, blue galaxies (van Zee 2001; Hunter \& Elmegreen 2004; Salzer et al. 2005). We estimated the SFR density of the ES/SDSS sample using the $u$-band luminosity as a proxy for SFR (Hopkins et al. 2003). We found that the SFR densities range from 0.0001 to 0.02 $M_{\odot} \mathrm{yr}^{-1} \mathrm{kpc}^{-2}$. This range is consistent with the SFR densities required to produce the blue offset in the galaxies in Figure 9. While the models might not be perfectly self-consistent as discussed above, they do demonstrate that strong emission lines can certainly affect the colors of galaxies. We note that the "pitched roof" shaped grids are a result of how the emission lines are affected by slight changes in the metallicity. It is clear that the span of these models can easily explain the galaxies that the stellar population models cannot and that many low-mass, blue galaxies must have colors that are dominated by emission lines (e.g., Zackrisson et al. 2005).

The emission-line effect may also amplify the underlying color offset discussed in the previous section by making galaxies appear bluer in $r-i$ than they actually are. However, the colors of the red galaxies in the offset region are dominated by their stellar populations and not likely affected by the emission-line colors.

\subsection{The Increase in Color Dispersion}

The analysis above demonstrates that the observed colors of the ES/SDSS sample can be well explained by stellar population models including emission lines, and that the general trend in

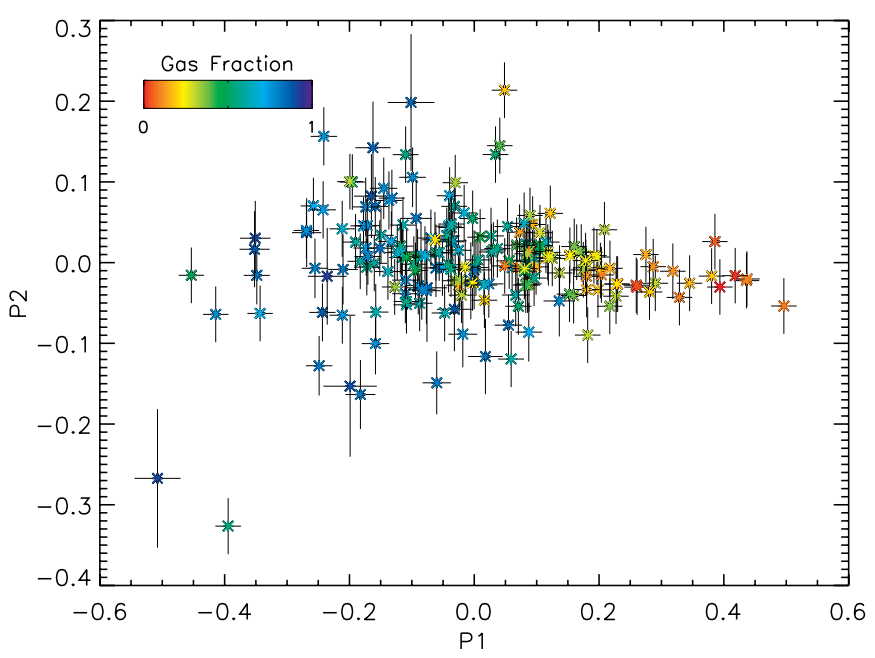

Figure 12. Principal axis of the galaxy color-color relation after PCA. Galaxies are color coded according to their gas fraction. Error bars were calculated using the uncertainties in the $g$-, $r$-, and $i$-band photometry.

(A color version of this figure is available in the online journal.)

color is due to decreasing mean stellar age with increasing gas fraction. However, while this explains most of the general trends seen in the color distribution in Figure 1, it does not fully explain the increase in the dispersion of color from red to blue galaxies. The narrow dispersion at red colors grows to a much larger value before the photometric uncertainties become large enough to be solely responsible for the discrepancy. Although some of the increased dispersion is likely due to the onset of emission-linedominated colors, this cannot account for the entirety of the color dispersion.

To explore how the dispersion in color relates to other physical quantities, we performed a principal component analysis (PCA) on the $r-i$ versus $g-r$ colors. The result aligns the principal axis $(P 1)$ with the galaxy color-color locus and sets the secondary axis $(P 2)$ perpendicular to $P 1 . P 1$ and $P 2$ can be expressed in term of the $g-r$ and $r-i$ colors of the galaxies by

$$
P 1=0.6(r-i)+0.8(g-r)-\langle 0.6(r-i)+0.8(g-r)\rangle
$$

and

$$
P 2=0.8(r-i)-0.6(g-r)-\langle 0.8(r-i)-0.6(g-r)\rangle,
$$

where the angle brackets indicate the mean of the enclosed quantity for the sample (the resulting eigenvalues are 6.2 and 0.8 for the eigenvectors pointing along the $P 1$ and $P 2$ axis, respectively).

Figure 12 shows the result of the PCA coordinate transformation. Error bars were calculated using the uncertainties in the $g_{-}$, $r$-, and $i$-band photometry. The $P 2$ axis serves as an indicator of dispersion from the galaxy locus and we compare the $P 2$ values to other physical parameters below.

We quantified the dispersion in the $P 2$ axis by calculating the rms $P 2$ scatter in bins of $P 1$. Figure 13 shows the resulting change in color dispersion along the principal axis. The error bars indicate the photometric uncertainty in the $P 2$ value. As seen in Figures 12 and 1, blue galaxies have a significantly larger color dispersion than red galaxies.

Changes in observable properties of the galaxies, such as stellar mass, rotation velocity, surface density, and surface brightness might be related to a change in their color dispersion. As seen in previous work on the ES/SDSS sample, many of 


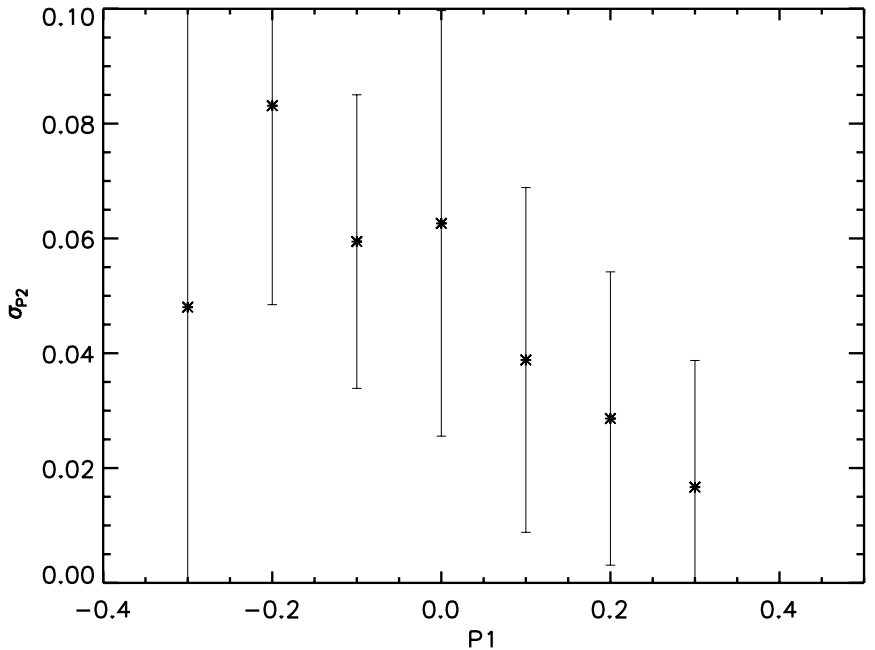

Figure 13. rms scatter in the $P 2$ axis as a function of $P 1$. The error bars indicate the combined photometric error of the data points in each bin.

these physical properties are related to each other (Disney et al. 2008; Garcia-Appadoo et al. 2009) and a change in one property is likely to show up as a variation in the others.

To examine how the change in color dispersion relates to the physical properties of the ES/SDSS galaxies, we plotted the P2 PCA values as a function of stellar mass, rotation velocity (measured from half of the $20 \% \mathrm{H}$ I velocity width), surface brightness, and stellar surface density (calculated by measuring the average stellar mass density within the $90 \%$ Petrosian radius). Figure 14 shows that changes in the physical properties trace the change in dispersion very well. In addition, Figure 14 has been color coded according to gas fraction. As seen in previous studies, the gas fractions also correlate with the physical properties of the ES/SDSS galaxies (Garcia-Appadoo et al. 2009). We quantified the change in color dispersion by plotting the rms scatter in the $P 2$ axis as a function of the four physical properties (Figure 15). All four properties correlate with the rms scatter in the $P 2$ variable. We computed the Spearman's rank correlation coefficient for each relation to test which property best correlates with the color dispersion. We calculated Spearman's $\rho$ values of $0.92,0.82,0.89$, and 0.96 for the stellar mass, velocity, surface brightness, and surface density, respectively. The stellar density shows the best correlation with color dispersion with the lowest density galaxies having the highest color dispersion.

The increase in color dispersion as a function of surface brightness and stellar surface density may probe a change in the star formation process. At the LSBs, the stochastic nature of star formation (Stinson et al. 2007), and the dominance of emission lines in low-mass galaxies, controls the observed colors and creates a larger color dispersion, while the colors of higher surface brightness systems are dominated by stellar populations (and to a smaller extent recent bursts of star formation, as discussed above). Because the surface brightness and stellar surface density are tied closely to other properties of the ES/SDSS galaxies (Disney et al. 2008), this change in color dispersion may be evidence for a significant change in the way stars form in galaxies.

While the stellar mass, surface brightness, and stellar surface density change smoothly, the velocity width shows a statistically significant $(4 \sigma)$ jump at a velocity of $80 \mathrm{~km} \mathrm{~s}^{-1}$. Previous studies have found that the rotation velocity in galaxies correlates with transitions in their disk properties. Dalcanton et al. (2004) showed that the presence of dust lanes in a sample of edge-on disk galaxies is related to rotation speed. At $V_{c}<120 \mathrm{~km} \mathrm{~s}^{-1}$, no dust lanes are present in their galaxies. Disk instabilities occurring at $V_{c}>120 \mathrm{~km} \mathrm{~s}^{-1}$ act to collapse
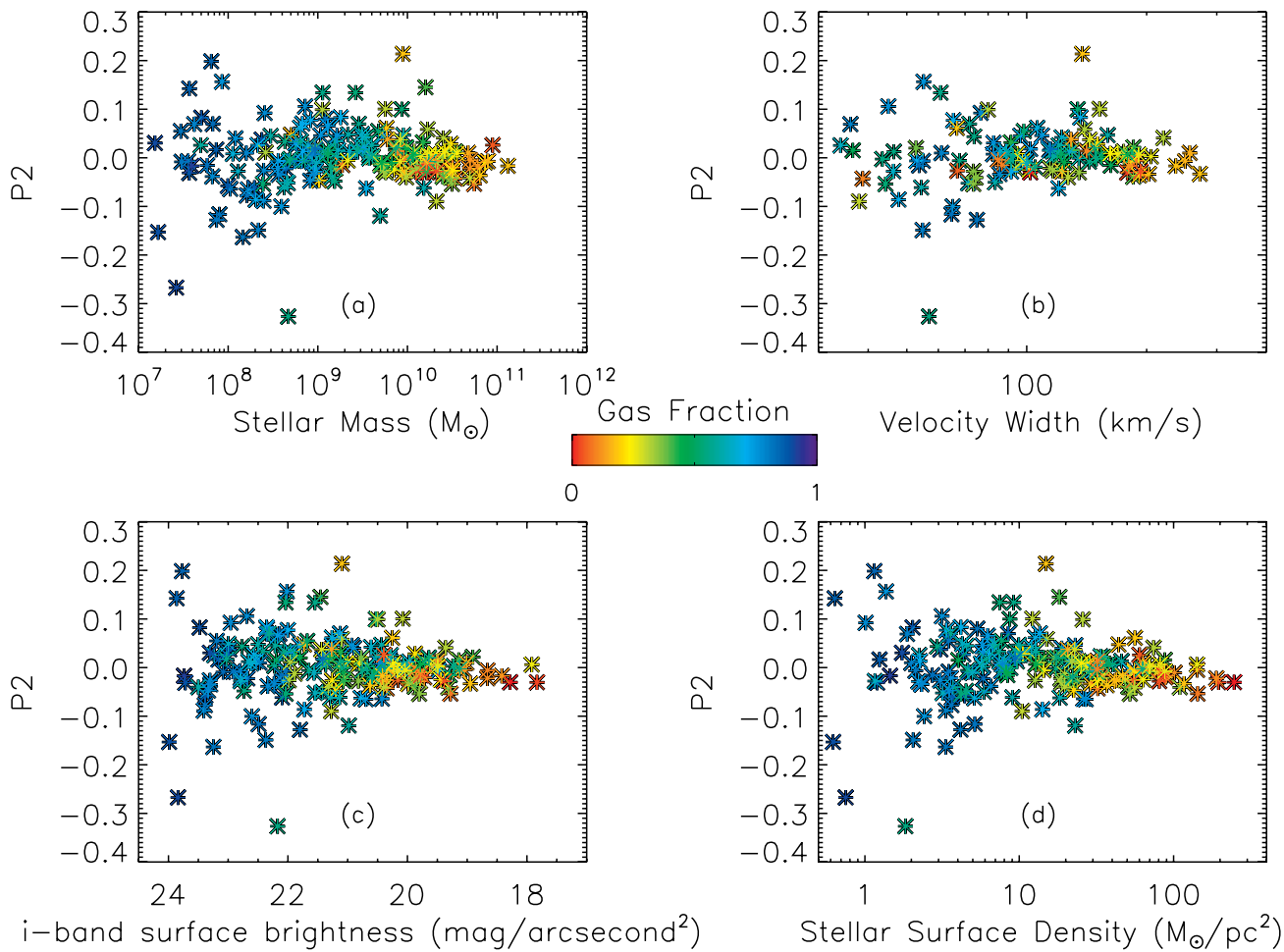

Figure 14. $P 2$ PCA axis as a function of stellar mass (a), velocity width (b), $i$-band surface brightness, (c) and stellar surface density (d). Points have been color coded according to their gas fractions.

(A color version of this figure is available in the online journal.) 

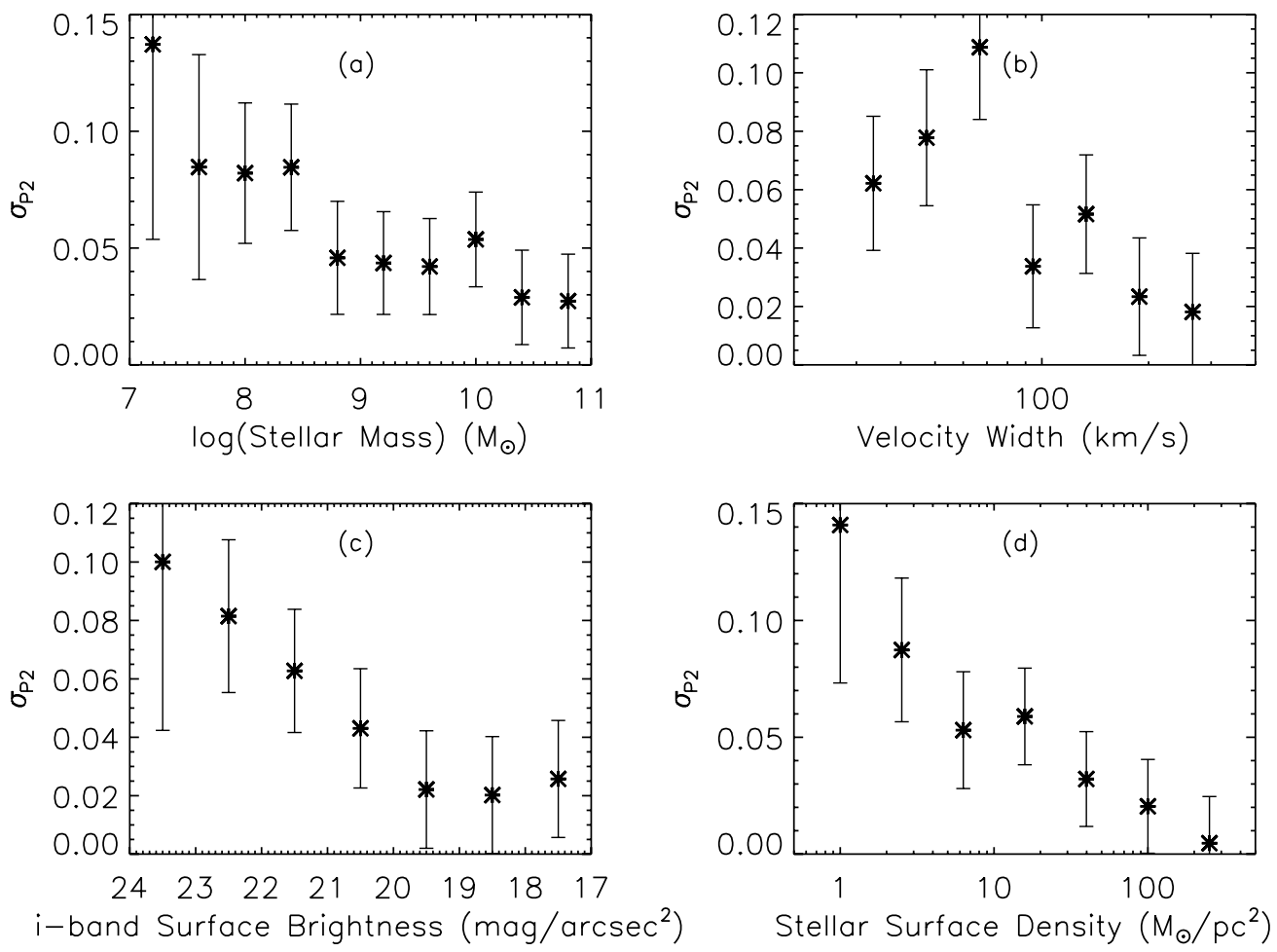

Figure 15. rms scatter in the $P 2$ axis as a function of stellar mass (a), velocity width (b), $i$-band surface brightness, (c) and stellar surface density (d). The error bars represent the combined photometric error in each bin.

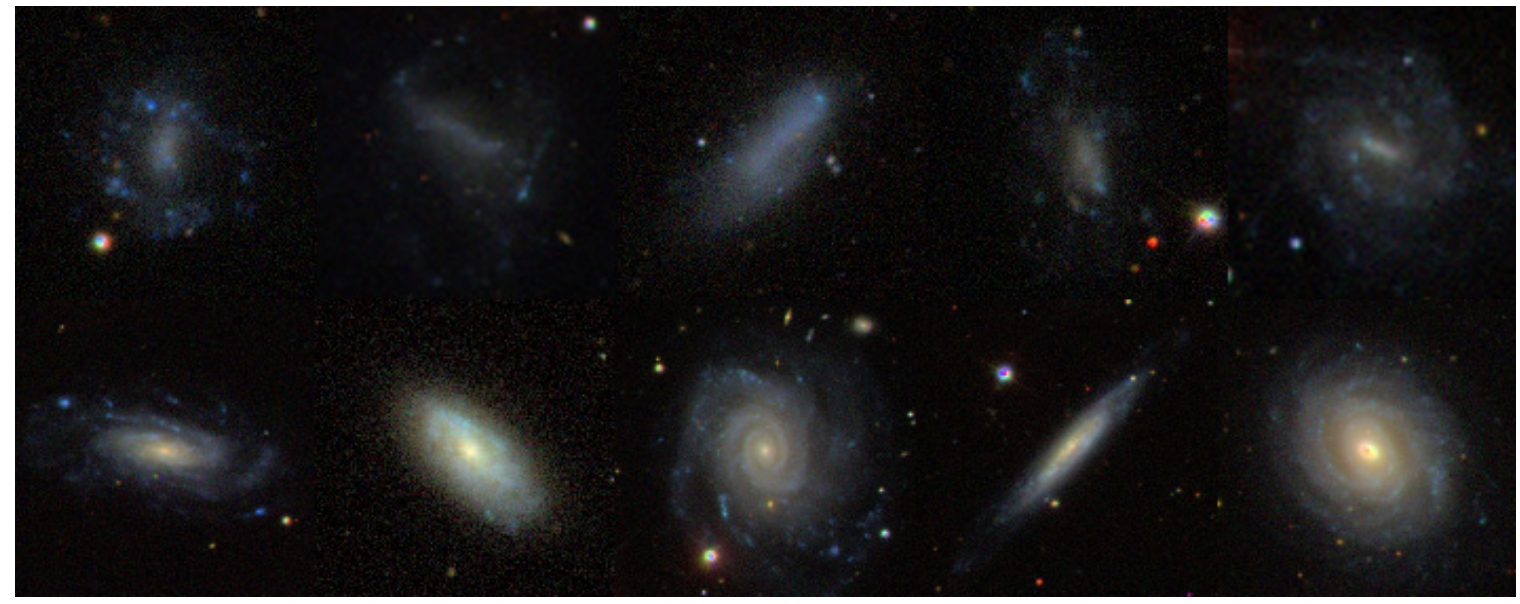

Figure 16. Five randomly selected gri composite images of galaxies from the slowly rotating regime $\left(V_{\text {rot }}<80 \mathrm{~km} \mathrm{~s}^{-1}\right.$; top row $)$ and five composite images from the faster regime ( $V_{\text {rot }}>80 \mathrm{~km} \mathrm{~s}^{-1}$; bottom row).

(A color version of this figure is available in the online journal.)

the dust to a small-scale height and produce a larger optical depth. Star formation efficiency is also increased above this velocity threshold, affecting the SFH and colors of the faster rotators (Verde et al. 2002; Dalcanton et al. 2004). Lee et al. (2007) find an additional transition around $50 \mathrm{~km} \mathrm{~s}^{-1}$, where the dispersion in $\mathrm{H} \alpha \mathrm{EW}$ dramatically increases. This transition is interpreted as a possible change from large-scale star formation in spiral galaxies to stochastic star formation in irregular systems (e.g., Stinson et al. 2007). We observe a transition in the color dispersion at $80 \mathrm{~km} \mathrm{~s}^{-1}$ (Figure 15(b)) that lies in between the Dalcanton and Lee transitions. The transition in the ES/SDSS sample may be a result of a similar physical process, namely, the change from well-organized star formation in massive disks to an irregular stochastic process in lower mass systems
To illustrate the differences between systems across the velocity transition, we randomly selected five gri composite images of galaxies from the low-velocity $\left(V_{\text {rot }}<80 \mathrm{~km} \mathrm{~s}^{-1}\right)$ population (top row of Figure 16) and five images from the highvelocity population $\left(V_{\mathrm{rot}}>80 \mathrm{~km} \mathrm{~s}^{-1}\right.$; bottom row of Figure 16). Figure 16 reveals a clear morphological distinction between the two populations, with the higher velocity systems having higher surface brightnesses, earlier morphological types, and a more organized distribution of stars (an indication of large-scale star formation).

\section{DISCUSSION}

We used the population synthesis models of Bruzual \& Charlot (2003) to model the SFHs and metallicities of galaxies 
in the ES/SDSS sample. We found that red galaxies have supersolar metallicities and have SFHs that are consistent with them forming the bulk of their stars in the distant past. These red galaxies have presumably exhausted their early gas supply but have recently acquired gas through mergers and infall. Although the infalling gas is likely low metallicity and could in principle dilute the metallicity, the small amount of gas in red galaxies (low gas fractions) is not enough to make an observable difference in the average metallicity of the ES/SDSS systems.

Bluer galaxies have lower metallicities and their mean stellar ages are younger (increasing $\tau$ values). The gas fractions suggest that these blue systems have at least as much gas as they do stars. Previous studies have indicated that these types of galaxies are less massive and have disks that are stable against collapse, making their star formation much less efficient. They have also likely retained much of their initial $\mathrm{H}$ I and are slowly converting it into stars as is suggested by the large (or negative) $\tau$ values. As galaxies pass the threshold for disk stability, their star formation becomes sporadic and there is a clear dispersion in the colors due to variations in burst age, burst strength, and superimposed emission lines. The idealistic Bruzual and Charlot models are likely only loose guides for these systems, as their SFHs no longer follow smooth exponential functions.

Figure 2 indicates that there are a few galaxies in the regime of exponentially increasing SFR (negative $\tau$ values). This is not surprising as there are few mechanisms that will increase the SFR of a galaxy. The likely culprit for these systems is the infall of gas. As gas is accreted, the gas densities in the galaxies will increase and local gravitational collapse will become more efficient. This will increase the SFR as well as the eventual metallicity of the galaxy, pushing its $g-r$ colors to the blue (more recent star formation) and its $r-i$ colors slightly to the red (more metals).

We also showed that $\mathrm{H}$ I-selected galaxies are offset from the SDSS galaxy locus, especially at the red end, and that this is likely due to bursts of star formation in the past few hundred Myr. The gas that induces these recent bursts is not primordial and is best explained by the accretion of gas-rich dwarfs.

The bluest galaxies in the ES/SDSS sample are not explained by population synthesis models alone. Their colors can be modeled only with the inclusion of emission lines. We showed that emission-line spectra with reasonable SFRs can explain the colors of the bluest galaxies in our sample.

We also showed that the distribution of galaxies at the red end of the color-color locus has a very small dispersion that continues to rise into the blue regime. This change in dispersion appears to correlate with stellar mass, rotation velocity, surface brightness, and especially stellar surface density. It is possible that the change in dispersion is evidence for a significant change in the way stars form in galaxies at a given mass scale. Massive galaxies have unstable disks and efficiently convert most of their primordial reservoirs of gas into stars in the first few Gyr after their formation. These galaxies are bulge dominated and have redder, older, and more massive populations of stars. We do see a sharp transition in the color distribution at a rotation velocity of $80 \mathrm{~km} \mathrm{~s}^{-1}$. This transition may be related to previous results that have found sharp transitions in galaxy properties as a function of rotational velocity (Dalcanton et al. 2004; Lee et al. 2007). However, further investigations are required to explore the various velocity transitions as a function of galaxy properties.

It may also be the case that the increase in dispersion is nothing more than a selection effect related to the surface brightness. As the surface brightness of a system decreases, the effect of a single burst of stars on the color becomes increasingly large, possibly explaining the increase in scatter. This effect might explain the onset of emission-line-dominated colors in the bluest galaxies as they can influence of the colors of galaxies devoid of massive stellar populations.

We note that the change in color dispersion is not easily seen in the volume-selected SDSS data. Applying the $\mathrm{H}$ I selection identifies a low dispersion subsample of the SDSS galaxies, most notably at the red end. As mentioned above, the red $\mathrm{H}$ I-selected galaxies appear to be bluer in $g-r$ than the "main" SDSS sample. These two features are likely related. It is possible that the large distribution in color at the red end of the SDSS main sample is due to the diversity of gas content. Because most of the red galaxies in SDSS exhausted their original supply of gas long ago, this dispersion may be an indication of the spread in time since the last major gas infall. We leave further discussion of the "color offset" to future study.

A.A.W. and J.J.D. acknowledge the support of NSF grant 0540567, the Royalty Research Fund, and ADVANCE. A.A.W. also acknowledges support from the Astronaut Scholarship Foundation. J.J.D. was partially supported by the Wyckoff Faculty Fellowship. Many thanks are extended to the anonymous referee for his/her many useful suggestions for improving this manuscript. The authors thank Mike Blanton, Marla Geha, Beth Willman, Paul Hodge, Suzanne Hawley, Tom Quinn, Peter Yoachim, Anil Seth, and Vandana Desai for useful discussions while completing this project. We also thank John Bochanski for technical assistance and Adam Burgasser for his financial support and insistence that this project reach completion.

Funding for the SDSS and SDSS-II has been provided by the Alfred P. Sloan Foundation, the Participating Institutions, the National Science Foundation, the U.S. Department of Energy, the National Aeronautics and Space Administration, the Japanese Monbukagakusho, and the Max Planck Society, and the Higher Education Funding Council for England. The SDSS Web site is http://www.sdss.org/.

The SDSS is managed by the Astrophysical Research Consortium (ARC) for the Participating Institutions. The Participating Institutions are the American Museum of Natural History, Astrophysical Institute Potsdam, University of Basel, University of Cambridge, Case Western Reserve University, The University of Chicago, Drexel University, Fermilab, the Institute for Advanced Study, the Japan Participation Group, The Johns Hopkins University, the Joint Institute for Nuclear Astrophysics, the Kavli Institute for Particle Astrophysics and Cosmology, the Korean Scientist Group, the Chinese Academy of Sciences (LAMOST), Los Alamos National Laboratory, the Max-PlanckInstitute for Astronomy (MPIA), the Max-Planck-Institute for Astrophysics (MPA), New Mexico State University, Ohio State University, University of Pittsburgh, University of Portsmouth, Princeton University, the United States Naval Observatory, and the University of Washington.

\section{REFERENCES}

Abazajian, K., et al. 2004, AJ, 128, 502

Abazajian, K., et al. 2009, ApJS, 182, 543

Adelman-McCarthy, J. K., et al. 2006, ApJS, 162, 38

Anders, P., \& Fritze-v. Alvensleben, U. 2003, A\&A, 401, 1063

Balcells, M., \& Peletier, R. F. 1994, AJ, 107, 135

Baldry, I. K., Glazebrook, K., Brinkmann, J., Ivezić, Ž., Lupton, R. H., Nichol, R. C., \& Szalay, A. S. 2004, ApJ, 600, 681 
Baldwin, J. A., Phillips, M. M., \& Terlevich, R. 1981, PASP, 93, 5

Ball, N. M., Loveday, J., Brunner, R. J., Baldry, I. K., \& Brinkmann, J. 2006, MNRAS, 373, 845

Barnes, D. G., et al. 2001, MNRAS, 322, 486

Bell, E. F., \& de Jong, R. S. 2000, MNRAS, 312, 497

Bell, E. F., \& de Jong, R. S. 2001, ApJ, 550, 212

Bell, E. F., McIntosh, D. H., Katz, N., \& Weinberg, M. D. 2003, ApJS, 149, 289

Bigiel, F., Leroy, A., Walter, F., Brinks, E., de Blok, W. J. G., Madore, B., \& Thornley, M. D. 2008, AJ, 136, 2846

Blanton, M. R., Eisenstein, D., Hogg, D. W., Schlegel, D. J., \& Brinkmann, J. 2005, ApJ, 629, 143

Blanton, M. R., et al. 2001, AJ, 121, 2358

Blanton, M. R., et al. 2003a, AJ, 125, 2348

Blanton, M. R., et al. 2003b, ApJ, 594, 186

Brinchmann, J., Charlot, S., White, S. D. M., Tremonti, C., Kauffmann, G., Heckman, T., \& Brinkmann, J. 2004, MNRAS, 351, 1151

Brooks, A. M., Governato, F., Quinn, T., Brook, C. B., \& Wadsley, J. 2009, ApJ, 694, 396

Bruzual, G., \& Charlot, S. 2003, MNRAS, 344, 1000

Chabrier, G. 2003, PASP, 115, 763

Covey, K. R., et al. 2007, AJ, 134, 2398

Dalcanton, J. J., \& Bernstein, R. A. 2002, AJ, 124, 1328

Dalcanton, J. J., Yoachim, P., \& Bernstein, R. A. 2004, ApJ, 608, 189

de Jong, R. S. 1996, J. Astron. Data, 2, 1

Disney, M. J., Romano, J. D., Garcia-Appadoo, D. A., West, A. A., Dalcanton, J. J., \& Cortese, L. 2008, Nature, 455, 1082

Dohm-Palmer, R. C., Skillman, E. D., Mateo, M., Saha, A., Dolphin, A., Tolstoy, E., Gallagher, J. S., \& Cole, A. A. 2002, AJ, 123, 813

Doyle, M. T., et al. 2005, MNRAS, 361, 34

Driver, S. P., et al. 2006, MNRAS, 368, 414

Fukugita, M., Ichikawa, T., Gunn, J. E., Doi, M., Shimasaku, K., \& Schneider, D. P. 1996, AJ, 111, 1748

Galaz, G., Dalcanton, J. J., Infante, L., \& Treister, E. 2002, AJ, 124, 1360

Garcia-Appadoo, D. A., West, A. A., Dalcanton, J. J., Cortese, L., \& Disney, M. J. 2009, MNRAS, 394, 340

Gavazzi, G., Bonfanti, C., Sanvito, G., Boselli, A., \& Scodeggio, M. 2002, ApJ, 576,135

Geha, M., Blanton, M. R., Masjedi, M., \& West, A. A. 2006, ApJ, 653, 240

Governato, F., Willman, B., Mayer, L., Brooks, A., Stinson, G., Valenzuela, O., Wadsley, J., \& Quinn, T. 2007, MNRAS, 374, 1479

Gunn, J. E., et al. 1998, AJ, 116, 3040

Gunn, J. E., et al. 2006, AJ, 131, 2332

Hambly, N. C., Davenhall, A. C., Irwin, M. J., \& MacGillivray, H. T. 2001a, MNRAS, 326, 1315

Hambly, N. C., Irwin, M. J., \& MacGillivray, H. T. 2001b, MNRAS, 326, 1295 Hambly, N. C., et al. 2001c, MNRAS, 326, 1279

Hogg, D. W., Finkbeiner, D. P., Schlegel, D. J., \& Gunn, J. E. 2001, AJ, 122, 2129

Hopkins, A. M., et al. 2003, ApJ, 599, 971

Hunter, D. A., \& Elmegreen, B. G. 2004, AJ, 128, 2170

Ivezić, Ž., et al. 2004, Astron. Nachr., 325, 583

Jarrett, T. H., Chester, T., Cutri, R., Schneider, S., Skrutskie, M., \& Huchra, J. P. 2000, AJ, 119, 2498

Johnson, B. D., et al. 2007, ApJS, 173, 392

Juneau, S., et al. 2005, ApJ, 619, L135

Kannappan, S. J. 2004, ApJ, 611, L89
Katz, N., Keres, D., Dave, R., \& Weinberg, D. H. 2003, in ASSL, 281, 185

Kauffmann, G., White, S. D. M., Heckman, T. M., Ménard, B., Brinchmann, J., Charlot, S., Tremonti, C., \& Brinkmann, J. 2004, MNRAS, 353, 713

Kauffmann, G., et al. 2003, MNRAS, 341, 33

Kewley, L. J., Dopita, M. A., Sutherland, R. S., Heisler, C. A., \& Trevena, J. 2001, ApJ, 556, 121

Lee, J. C., Kennicutt, R. C., Funes José, G. S. J., Sakai, S., \& Akiyama, S. 2007, ApJ, 671, L113

Leroy, A. K., et al. 2009, AJ, 137, 4670

Lupton, R. H., Ivezic, Z., Gunn, J. E., Knapp, G., Strauss, M. A., \& Yasuda, N. 2002, in SPIE Conf. Ser. 4836, ed. J. A. Tyson \& S. Wolff (Bellingham, WA SPIE), 350

MacArthur, L. A., Courteau, S., Bell, E., \& Holtzman, J. A. 2004, ApJS, 152, 175

Magris, C. G., Binette, L., \& Bruzual, A. G. 2003, ApJS, 149, 313

Maller, A. H., Berlind, A. A., Blanton, M. R., \& Hogg, D. W. 2009, ApJ, 691, 394

Matthews, L. D., Gao, Y., Uson, J. M., \& Combes, F. 2005, AJ, 129, 1849

McQuinn, K. B. W., Skillman, E. D., Cannon, J. M., Dalcanton, J. J., Dolphin, A., Stark, D., \& Weisz, D. 2009, ApJ, 695, 561

Meyer, M. J., et al. 2004, MNRAS, 350, 1195

Oke, J. B., \& Gunn, J. E. 1983, ApJ, 266, 713

Pier, J. R., Munn, J. A., Hindsley, R. B., Hennessy, G. S., Kent, S. M., Lupton, R. H., \& Ivezić, Ž. 2003, AJ, 125, 1559

Pizagno, J., et al. 2005, ApJ, 633, 844

Roberts, M. S., \& Haynes, M. P. 1994, ARA\&A, 32, 115

Rosenberg, J. L., Schneider, S. E., \& Posson-Brown, J. 2005, AJ, 129, 1311

Salim, S., et al. 2005, ApJ, 619, L39

Salzer, J. J., Jangren, A., Gronwall, C., Werk, J. K., Chomiuk, L. B., Caperton, K. A., Melbourne, J., \& McKinstry, K. 2005, AJ, 130, 2584

Sarajedini, A., Brandt, K., Grocholski, A. J., \& Tiede, G. P. 2004, AJ, 127, 991

Schlegel, D. J., Finkbeiner, D. P., \& Davis, M. 1998, ApJ, 500, 525

Schombert, J. M., Bothun, G. D., Impey, C. D., \& Mundy, L. G. 1990, AJ, 100, 1523

Searle, L., Sargent, W. L. W., \& Bagnuolo, W. G. 1973, ApJ, 179, 427

Skibba, R. A., et al. 2008, arXiv:0811.3970

Skrutskie, M. F., et al. 2006, AJ, 131, 1163

Smith, J. A., et al. 2002, AJ, 123, 2121

Stinson, G. S., Dalcanton, J. J., Quinn, T., Kaufmann, T., \& Wadsley, J. 2007, ApJ, 667, 170

Stoughton, C., et al. 2002, AJ, 123, 485

Strateva, I., et al. 2001, AJ, 122, 1861

Tinsley, B. M. 1972, A\&A, 20, 383

Tinsley, B. M., \& Gunn, J. E. 1976, ApJ, 203, 52

Tremonti, C. A., et al. 2004, ApJ, 613, 898

Tully, R. B., Pierce, M. J., Huang, J.-S., Saunders, W., Verheijen, M. A. W., \& Witchalls, P. L. 1998, AJ, 115, 2264

van Zee, L. 2001, AJ, 121, 2003

Verde, L., Oh, S. P., \& Jimenez, R. 2002, MNRAS, 336, 541

West, A. A., Garcia-Appadoo, D. A., Dalcanton, J. J., Disney, M. J., Rockosi, C. M., Ivezić, Ž., Bentz, M. C., \& Brinkmann, J. 2009, AJ, submitted

Wong, O. I., et al. 2006, MNRAS, 371, 1855

York, D. G., et al. 2000, AJ, 120, 1579

Zackrisson, E., Bergvall, N., \& Leitet, E. 2008, ApJ, 676, L9

Zackrisson, E., Bergvall, N., Olofsson, K., \& Siebert, A. 2001, A\&A, 375, 814

Zackrisson, E., Bergvall, N., \& Östlin, G. 2005, A\&A, 435, 29

Zwaan, M. A., et al. 2004, MNRAS, 350, 1210 